\title{
Postoperative Pneumonia Following Open Heart Surgery
}

Omar A. Alsulami ${ }^{1}$, Abdulhadi E. Konkar ${ }^{1}$, Abdulrahman A. Alalyani ${ }^{1}$, Muath S. Alghamdi ${ }^{1}$, Siraj M. Eid ${ }^{1}$ , Hazem A. Alsulami ${ }^{2}$, Khalid E. Al-Ebrahim ${ }^{3}$

1. Medicine, King Abdulaziz University, Jeddah, SAU 2. Pharmacy, King Abdulaziz University, Jeddah, SAU 3. Surgery, King Abdulaziz University, Jeddah, SAU

Corresponding author: Omar A. Alsulami, dr.3mrsa@gmail.com

\section{Abstract \\ Objectives}

This study aimed to measure the incidence and record the relations between risk factors of postoperative pneumonia (POP) among patients who underwent open heart surgery in a single hospital in Saudi Arabia.

\section{Methods}

This retrospective cohort study was conducted in June 2019 at King Abdulaziz University hospital in Saudi Arabia. Data including general information, comorbidities, lab investigations, preoperative risk factors, intraoperative considerations, and postoperative elements were collected and analyzed.

\section{Results}

A total of 255 cardiac surgeries were performed from November 2014 to June 2019. Two hundred of the 255 cardiac surgeries were analyzed as open-heart surgeries. Only five patients were diagnosed with POP after open heart surgery with an incidence of $2.5 \%$. The mean age of these patients was $47 \pm 18$ years, more than half of them were smokers, three were hypertensive, four were classified as ASA 4, and three underwent the operation electively. The mean bypass time was $100.3 \pm 24.5 \mathrm{~min}$, the mean duration of operation was $199 \pm$ $86.2 \mathrm{~min}$, the mean postoperative intensive care unit (ICU) stay was $97.4 \pm 83.4$ hours, and the mean overall hospital stay was $10.4 \pm 7.2$ days. We observed significant differences in only the following correlations: amount of blood transfusion with ICU stay and with the overall hospital stay.

\section{Conclusion}

The incidence of developing postoperative pneumonia in patients undergoing open heart surgery in the King Abdulaziz University hospital from November 2014 to June 2019 was $2.5 \%$, indicating a high-quality level of surgical technique and proper infection control.

Received 08/18/2020

Review began 08/19/2020 Review ended 09/04/2020 Published 09/08/2020

๑) Copyright 2020

Alsulami et al. This is an open access article distributed under the terms of the Creative Commons Attribution License CC-BY 4.0., which permits unrestricted use, distribution, and reproduction in any medium, provided the original author and source are credited.
Categories: Cardiac/Thoracic/Vascular Surgery, Infectious Disease

Keywords: cardiac surgery, pneumonia, postoperative pneumonia, hospital acquired pneumonia, operative complication, surgery, infection control

\section{Introduction}

Respiratory complications following cardiac surgeries are one of the most important concerns for cardiac surgeons and anesthesiologists [1,2]. However, pneumonia is ranked as the second most common nosocomial infection after urinary tract infections and approximately half of the cases occur postoperatively [3]. In fact, it is considered the third most common postoperative complication [4]. Postoperative pneumonia (POP) can be defined as either hospital-acquired pneumonia (pneumonia developing $48-72 \mathrm{~h}$ after admission) or ventilator-associated pneumonia (VAP) (pneumonia developing 48-72 $\mathrm{h}$ after endotracheal intubation) occurring in a post-surgical patient [4]. Although there have been many advances in surgery and anesthesia, POP continues to be a significant problem [5]. In an observational study of 16084 patients undergoing coronary artery bypass grafting (CABG), postoperative pneumonia was found in 531 (3.30\%) cases [6]. Furthermore, previous studies recorded POP as the most common infection after cardiac surgery (CS) with a prevalence rate between $2 \%$ and $10 \%$, especially during the first postoperative week [7-10].

Several studies have reported the incidence rates and causal factors associated with POP across the world. However, few studies have reported the incidence of POP after cardiac surgery in Saudi Arabia. Therefore, our aim in this study was to measure the incidence and record the relations between risk factors of POP among patients who underwent open heart surgery between November 2014 and June 2019 at our institute.

\section{Materials And Methods}


This retrospective study was conducted in June 2019 in the surgery department of a single institute in Saudi Arabia. The medical records of all the patients who underwent cardiac surgery during the period from November 2014 to June 2019 were initially examined. Patients who required mechanical ventilation before surgery, experienced death before the second postoperative day, had a history of lung transplantation, or who did not undergo open heart surgery with cardiopulmonary bypass were excluded from the study. Ethical approval for this study was obtained from our Institutional Review Board (IRB). Informed consent was waived due to the retrospective nature of the study.

Data collected from the medical records was divided into 6 parts: (1) general information, such as medical record number, age, gender, history of smoking, and body mass index (calculated by weight in $\mathrm{Kg} / \mathrm{height}$ in $\mathrm{cm}^{2}, \mathrm{BMI} \geqslant 30$ were considered obese); (2) comorbidities, such as immunodeficiency, hypertension, chronic obstructive pulmonary disease (COPD), and diabetes mellitus (DM); (3) lab investigations including culture results, RBC count, WBC count, and platelet count; (4) preoperative risk factors, including the type of operation (elective, redo, urgent) and American Society of Anesthesiologists (ASA) Classification (ASA 1: Healthy patient, ASA 2: mild systemic disease, ASA 3: moderate systemic disease, ASA 4: severe systemic disease, ASA 5: moribund patient); (5) intraoperative considerations, such as the amount of blood transfusion (in ml), antibiotic prophylaxis (by dose), duration of operation (in min), duration of cardiopulmonary bypass (in min), and site of the incision; and (6) postoperative elements, including the patient temperature (in Celsius) and overall hospital stay.

Postoperative pneumonia in non-intubated patients was diagnosed by the presence of a postoperative increase in temperature, a positive sputum culture, or new lung opacity on an x-ray film. Regarding VAP, Ventilator-Associated Events developed by the Centers for Disease Control and Prevention (CDC) were used as criteria to diagnose POP. Infection-related ventilator-associated complications (IVAC) were diagnosed by a ventilator-associated condition (VAC), which is an increase in oxygen requirement $(\geqslant 0.20$ in the fraction of inspired oxygen: $\mathrm{FiO} 2$ ) or positive end-expiratory pressure (PEEP; $\geqslant 3 \mathrm{~cm} \mathrm{H} 2 \mathrm{O})$ after a period of stability $(\geqslant 2$ days), in addition to a temperature greater than $38^{\circ} \mathrm{C}$ or less than $36^{\circ} \mathrm{C}$ or a white blood cell count greater than $12,000 \mathrm{~mm}^{3}$ or less than $4,000 \mathrm{~mm}^{3}$, and a new antibiotic administered for at least four days. In addition to the above, the existence of purulent secretions or positive respiratory cultures (regardless of chest film findings) indicated possible or probable VAP.

Data were analyzed and entered by the statistical package for social sciences (SPSS) version 21. Continuous variables were described by calculating the standard deviation and mean. We calculated frequencies to define categorical variables. An independent T-test was used to evaluate the difference between qualitative and quantitative variables (the difference in the mean of bypass times, ICU stays, and duration of operation between POP and non-POP patients). The Chi-square test was used to evaluate the relationship between two qualitative variables (POP versus non-POP patients with smoking, categorical BMI, and temperature coded as a nominal variable). A correlation analysis was used to determine relationships between two quantitative variables (ICU length of stay with the amount of blood transfusion, and body temperature with ICU length of stay). Tests that yielded a P-value $<0.05$ were considered significant.

\section{Results}

From November 2014 to June 2019, a total of 255 cardiac open heart surgeries were performed in our institute. Of these, we excluded 55 cases that did not undergo cardiopulmonary bypass (10 due to wound infection, eight due to mediastinal lesions, six due to transthoracic pericardial infusion drainage, five due to removal of the mediastinal wire, and 16 did not have operation data). Finally, we enrolled a total of 200 patients (age: $14-84$ years; male: $\mathrm{n}=158,79 \%$ ) in this study.

Patient demographics are presented in Table 1. Regarding all participants, $164(82 \%)$ of the surgeries were elective operations, 172 (86\%) of the patients were classified as ASA 4, 81 (40.5\%) were smokers, 111 (55.5\%) were hypertensive, 199 (99.5\%) had not suffered any immunodeficiency, 192 (96\%) were afebrile, and $42 \%$ of them did not receive blood while $31 \%$ were given one unit. According to their body mass index, 77 (38.5\%) of the participants were overweight. The mean duration of operation for all participants was $246.6 \pm 67.9 \mathrm{~min}$ and the mean overall hospital stay was $11.8 \pm 13.7$ days. 


\section{Cureus}

Demographic data and predictive factors of patients

AGE

More than 60

30-59

Less than 30

9

Gender

Male

Female

Starved

Normal

BMI

Over-weight

Obese 1

Obese 2

Obese 3

History of smoking

Smoker

0

Amount of blood transfusion (ml)

Classification of Operation

ASA2

(ASA) Classification

ICU Stay

\section{TABLE 1: Demographic data and predictive factors of patients}

BMI (body mass index), ASA (American society of anesthesiologist), ICU (Intensive care unit), CPB (cardiopulmonary bypass). 


\section{Cureus}

We found that 19 (9.5\%) of the participants had a positive culture (Table 2). Among them, 6 (3\%) had Haemophilus influenzae; only five of the 19 had POP and most of them ( 3 cases) also had Haemophilus influenzae. Figure 1 shows the antibiotic prophylaxis of all the patients

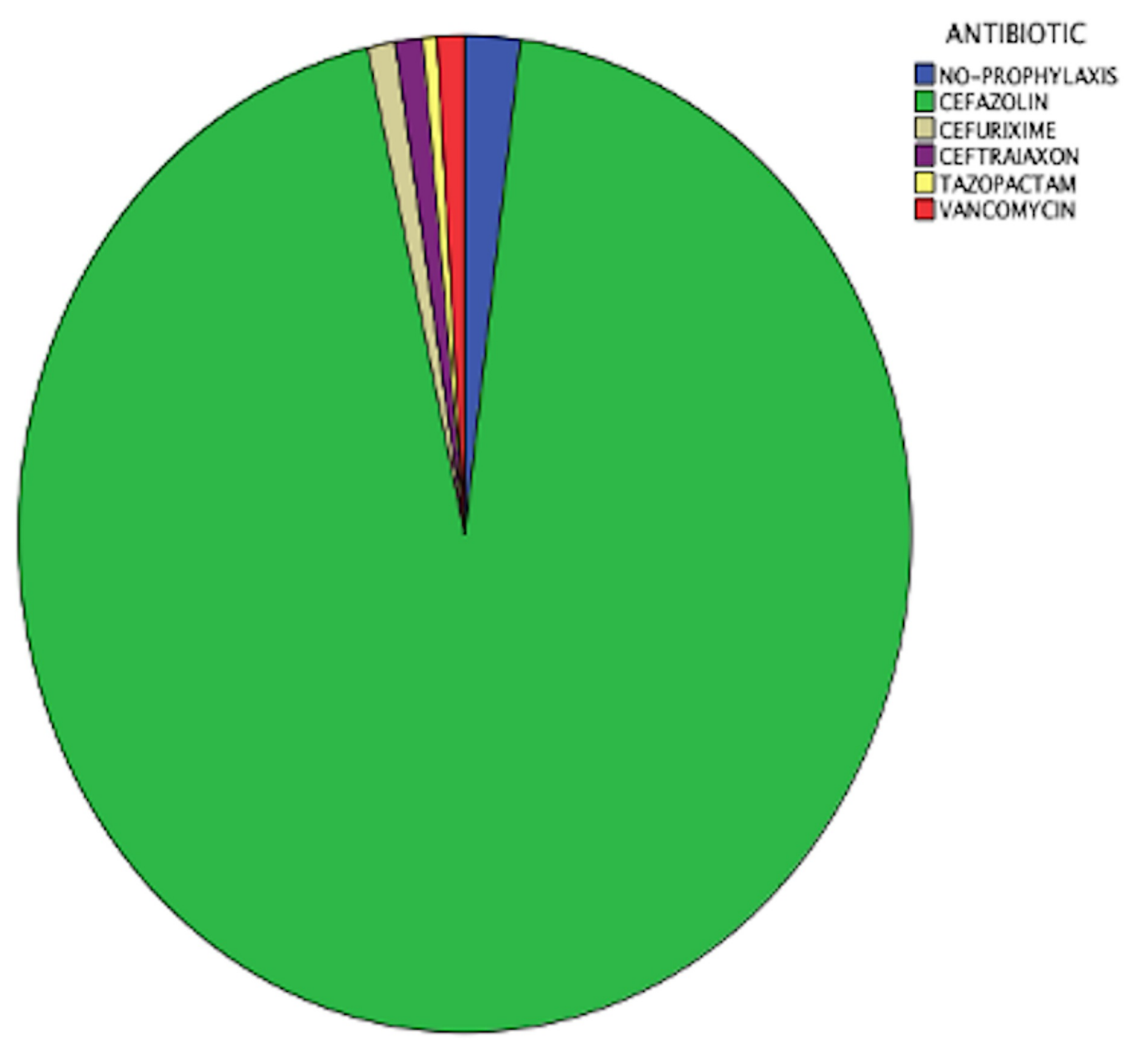

FIGURE 1: antibiotic prophylaxis

Culture results

Count

Normal upper respiratory tract flora

3

Haemophilus influenzae

6

Klebsiella

1

Pseudomonas aeruginosa

1

Diphtheroid species

1

Gram-negative-bacilli

2

mixed-bacteria

2

Coagulase negative Staphylococcus

1

Enterobacter cloacae

1

yeast cells

1

\section{TABLE 2: Culture results}

Finally, we diagnosed only five of the 200 patients with POP after open heart surgery with an incidence of $2.5 \%$. Their mean age was $47 \pm 18$ years, more than half of them were smokers, three were hypertensive, four 
were classified as ASA 4, and three underwent the operation electively. The mean bypass time was $100.3 \pm 24.5 \mathrm{~min}$, the mean of duration of operation was $199 \pm 86.2 \mathrm{~min}$, the mean postoperative intensive care unit (ICU) stay was $97.4 \pm 83.4$ hours, and the mean overall hospital stay was $10.4 \pm 7.2$ days. There was a significant difference between the overall hospital stay and duration of operation $(p=0.033$, Pearson correlation= 0.142$)$. Three of the five cases received one unit $(450 \mathrm{ml})$ of blood during the operation, and cefazolin was administrated as prophylaxis to all of them with a dose equal to $2000 \mathrm{mg}$ for 3 of them; all five cases were afebrile directly after the operation.

\section{Discussion}

Due to advances in medical technology, the mortality rate following cardiac operations has decreased substantially. However, the incidence of postoperative pneumonia varies widely, which may be explained by differences in infection control levels between different hospitals and/or differences in the criteria used for diagnosis between researchers. Hence, the purpose of this retrospective study was to evaluate the incidence of postoperative pneumonia in a Saudi Arabian hospital.

According to the literature, risk factors associated with postoperative pneumonia after open heart surgery include old age (> 50 years), gender, body mass index, history of smoking, American society of anthologists classification of more than 2 (ASA 2), CPB time, amount of blood transfusion, classification of operation, duration of operation, ICU stay, and overall hospital stay [7-10]. We analyzed all of these risk factors in this study and importantly, only found significance in the following correlations: amount of blood transfusion with ICU stay and with the overall hospital stay.

Besides surgical technique, age is considered as a risk factor for POP following cardiac surgery [11-15]. In 2017, a retrospective study conducted in Japan which included 123 elderly patients ( $>65$ years old) who underwent cardiac surgery found that $9.8 \%$ suffered from POP [16]. However, in our study analysis, there were a total of 115 patients who were considered elderly, but only two out the five patients diagnosed with POP were elderly. Therefore, our study does not support this point.

A 2012 cohort study conducted in Saudi found that most POP patients were young males with lower admission severity of illness scores such as the Glasgow coma scale [17]. Similarly, in our study, we found that all five cases diagnosed with postoperative pneumonia were male. However, an American study states that females are at a higher risk for postoperative pneumonia [18]. Another observational study reported that gender and body mass index did not significantly increase a patient's risk of developing pneumonia [6]. In the body mass index classification we employed, most of the postoperative pneumonia patients in our study were not obese $n=4$ (normal weight $=2$, overweight $=2$ ). On the other hand, a study by Likosky et al. reported that patients with pneumonia were more likely to have greater body mass index [19].

In our study, four out of five POP patients had a history of smoking; this disagrees with a previous study conducted with 5158 POP patients which reported that a history of smoking did not increase the risk of pneumonia [20]. Another risk factor related to POP is chronic obstructive pulmonary disease (COPD). A retrospective study in Turkey reported that COPD may cause POP. [12] In our study, there were no cases of COPD among patients diagnosed with POP. Nonetheless, a previous retrospective study reported that older age and lung disease were shown to increase the risk of developing postoperative pneumonia following cardiac surgery. [21]

Our findings are consistent with other studies, which have shown that patients with an ASA classification $>2$ are considered at risk for developing postoperative pneumonia. [22] Indeed, we noted that all five POP cases in our study were classified as either ASA 4 and ASA 3.

The most commonly isolated microorganism in this study was Haemophilus influenzae. However, Allou et al. found that Enterobacteriaceae is the most commonly isolated organism, followed by non-fermenting Gram-negative bacilli. Another French study conducted on 1589 patients found that the most isolated micro-organism was Staphylococcus aureus [23].

In our research, there was significance between the overall hospital stay and the duration of operation. This suggests that an increase in the duration of the operation will lengthen the overall hospital stay $(\mathrm{p}=0.033$, Pearson correlation= 0.142 ). A prospective study in Boston revealed that intraoperative events (amount of blood transfused and operative time) have a statistically significant impact on the overall hospital stay [24]. Likewise, in our study, we observed a significant relationship between the overall hospital stay and the amount of PRBCs transfused intra-operatively ( $\mathrm{p}=0.009$, Pearson correlation= 0.184 ).

A prospective study conducted in Spain found that blood transfusion may cause transient immune suppression. Hence, a blood transfusion may increase the risk of developing POP [25]. Additionally, an American study of 16182 patients conducted in 2015 reported a dependent relationship between the number of RBC units transfused and the odds of pneumonia [20]. In 2006, a prospective study reported that transfused patients had higher rates of intensive care unit (ICU) stay compared to those who did not receive a blood transfusion [26]. In our study analysis, we found that patients receiving blood transfusions had a 
In this study, we found that there was a significant relationship between the length of stay at the ICU and elevated body temperature. Thus, an increase in body temperature will lead to a prolonged duration of stay in the ICU.

A limitation of our study concerning postoperative pneumonia after open heart surgery is the lack of studies conducted in our country, which presented unexpected challenges. The study design was a retrospective study performed in one center, which creates problems, such as small population, missing data, and limited access to the database. We recommend future studies with a prospective, multicenter design to recruit a larger population for a more extended period. Moreover, the study should cover all cardiac procedures instead of only open heart surgeries. Furthermore, we advise studying the relation between the amount of blood transfused and the postoperative stay at ICU as statistics showed a directly proportional relationship between those two factors ( $\mathrm{P}=0.048$ Pearson correlation=0.140).

\section{Conclusions}

In conclusion, we found that the incidence of developing postoperative pneumonia among open heart surgery patients in our institute between 2014 and 2019 was $2.5 \%$, indicating a high-quality level of surgical technique and proper infection control. Haemophilus influenzae represent the most common cause in our observations.

\section{Additional Information \\ Disclosures}

Human subjects: All authors have confirmed that this study did not involve human participants or tissue. Animal subjects: All authors have confirmed that this study did not involve animal subjects or tissue. Conflicts of interest: In compliance with the ICMJE uniform disclosure form, all authors declare the following: Payment/services info: All authors have declared that no financial support was received from any organization for the submitted work. Financial relationships: All authors have declared that they have no financial relationships at present or within the previous three years with any organizations that might have an interest in the submitted work. Other relationships: All authors have declared that there are no other relationships or activities that could appear to have influenced the submitted work.

\section{References}

1. Montes FR, Maldonado JD, Paez S, Ariza F: Off-pump versus on-pump coronary artery bypass surgery and postoperative pulmonary dysfunction. J Cardiothorac Vasc Anesth. 2004, 18:698-703. 10.1053/j.jvca.2004.08.004

2. Roger VL, Go AS, Lloyd-Jones DM, et al.: Heart disease and stroke statistics--2011 update: a report from the American Heart Association. Circulation. 2011, 123:18-209. 10.1161/CIR.0b013e3182009701

3. Fujita T, Sakurai K: Multivariate analysis of risk factors for postoperative pneumonia . Am J Surg. 1995, 169:304-307. 10.1016/S0002-9610(99)80163-9

4. Kazaure HS, Martin M, Yoon JK, Wren SM: Long-term results of a postoperative pneumonia prevention program for the inpatient surgical ward. JAMA Surg. 2014, 149:914-918. 10.1001/jamasurg.2014.1216

5. Chughtai M, Gwam CU, Mohamed N, et al.: The epidemiology and risk factors for postoperative pneumonia . J Clin Med Res. 2017, 9:466-475. 10.14740/jocmr3002w

6. Strobel RJ, Liang Q, Zhang M, et al.: A pre-operative risk model for post-operative pneumonia folowing coronary artery bypass grafting. Ann Thorac Surg. 2016, 102:1213-1219. 10.1016/j.athoracsur.2016.03.074

7. El Solh AA, Bhora M, Pineda L, Dhillon R: Nosocomial pneumonia in elderly patients following cardiac surgery. Respir Med. 2006, 100:729-736. 10.1016/j.rmed.2005.07.011

8. Hortal J, Giannella M, Pérez MJ, et al.: Incidence and risk factors for ventilator-associated pneumonia after major heart surgery. Intensive Care Med. 2009, 35:1518-1525. 10.1007/s00134-009-1523-3

9. Hortal J, Muñoz P, Cuerpo G, Litvan H, Rosseel PM, Bouza E: Ventilator-associated pneumonia in patients undergoing major heart surgery: an incidence study in Europe. Crit Care. 2009, 13:80. 10.1186/cc7896

10. Kollef MH, Sharpless L, Vlasnik J, Pasque C, Murphy D, Fraser VJ: The impact of nosocomial infections on patient outcomes following cardiac surgery. Chest. 1997, 112:666-675. 10.1378/chest.112.3.666

11. Bicer Y, Simsek S, Yapici N, Aydin O, Sogut F, Aykac Z: Risk factor analysis of pneumonias developing after open heart surgery. Crit Care. 2005, 9:10. 10.1186/cc3073

12. Kinlin LM, Kircher C, Huiling Z, Daley J, Fisman DN: Derivation and validation of a clinical prediction rule for nosocomial pneumonia after coronary artery bypass graft surgery. Clin Infect Dis. 2010, 50:493-501. 10.1086/649925

13. Puzio J, Kucewicz E, Siola M, et al.: Atypical and opportunistic pulmonary infections after cardiac surgery . Anestezjol Intens Ter. 2009, 41:41-45.

14. Fukui T, Manabe S, Shimokawa T, Takanashi S: Incidence and outcomes of pneumonia after isolated offpump coronary artery bypass grafting. Heart Surg Forum. 2009, 12:194-198.

15. Vamvakas EC, Carven JH: Exposure to allogeneic plasma and risk of postoperative pneumonia and/or wound infection in coronary artery bypass graft surgery. Transfusion. 2002, 42:107-113. 10.1046/j.15372995.2002.00001.x

16. Miyata E, Tanaka A, Emori H, Taruya A, Miyai S, Sakagoshi N: Incidence and risk factors for aspiration pneumonia after cardiovascular surgery in elderly patients. Gen Thorac Cardiovasc Surg. 2017, 65:96-101. 


\section{Cureus}

10.1007/s11748-016-0710-8

17. Al-Dorzi HM, El-Saed A, Rishu AH, Balkhy HH, Memish ZA, Arabi YM: The results of a 6-year epidemiologic surveillance for ventilator-associated pneumonia at a tertiary care intensive care unit in Saudi Arabia. Am J Infect Control. 2012, 40:794-799. 10.1016/j.ajic.2011.10.004

18. Gelijns AC, Moskowitz AJ, Acker MA, et al.: Management practices and major infections after cardiac surgery. J Am Coll Cardiol. 2014, 64:372-381. 10.1016/j.jacc.2014.04.052

19. Likosky DS, Paone G, Zhang M, et al.: Red blood cell transfusions impact pneumonia rates after coronary artery bypass grafting. Ann Thorac Surg. 2015, 100:794-801. 10.1016/j.athoracsur.2015.03.089

20. Ailawadi G, Chang HL, O'Gara PT, et al.: Pneumonia after cardiac surgery: experience of the National Institutes of Health/Canadian Institutes of Health Research Cardiothoracic Surgical Trials Network. J Thorac Cardiovasc Surg. 2017, 153:1384-1391. 10.1016/j.jtcvs.2016.12.055

21. Topal AE, Eren MN: Risk factors for the development of pneumonia post cardiac surgery . Cardiovasc J Afr. 2012, 23:212-215. 10.5830/CVJA-2012-005

22. Kodra N, Shpata V, Ohri I: Risk factors for postoperative pulmonary complications after abdominal surgery . Maced J Med Sci. 2016, 4:259-263. 10.3889/oamjms.2016.059

23. Legras A, Malvy D, Quinioux AI, Villers D, Bouachour G, Robert R, Thomas R: Nosocomial infections: prospective survey of incidence in five French intensive care units. Intensive Care Med. 1998, 24:1040-1046. 10.1007/s001340050713

24. Collins TC, Daley J, Henderson WH, Khuri SF: Risk factors for prolonged length of stay after major elective surgery. Ann Surg. 1999, 230:251. 10.1097/00000658-199908000-00016

25. Tamayo E, Álvarez FJ, Martínez-Rafael B, et al.: Ventilator-associated pneumonia is an important risk factor for mortality after major cardiac surgery. J Crit Care. 2012, 27:18-25. 10.1016/j.jcrc.2011.03.008

26. Taylor RW, O’Brien J, Trottier SJ, et al.: Red blood cell transfusions and nosocomial infections in critically ill patients. Crit Care Med. 2006, 34:2302-2308. 10.1097/01.CCM.0000234034.51040.7F 